\title{
Pneumococcal septic arthritis in rheumatoid arthritis
}

\author{
PETER K MORLEY, RICHARD G HULL, AND M ANN HALL \\ From the Rheumatology Unit, Wexham Park Hospital, Slough, Berks
}

SUMMARY Septic arthritis is associated with a definite morbidity which may be related to a delay in diagnosis and hence treatment. The cases of three patients with rheumatoid arthritis and ${ }_{\sigma}^{\omega}$ chronic chest disease where the joint sepsis was not the predominant feature are presented. The $\frac{\varrho}{2}$ responsible organism was Streptococcus pneumoniae which had spread after recent chest infections. Minimal joint symptoms or general malaise in association with an unexplained rise in $\%$ erythrocyte sedimentation rate in these circumstances warrant a search for joint sepsis.

Key words: Streptococcus pneumoniae.

A septic joint usually presents as an acutely painful warm effusion with loss of function. In the presence of an inflammatory arthritis the signs may not be so prominent, but pain in the affected joint is still a feature. In addition, sepsis may follow local trauma, intra-articular corticosteroids, and surgery. A successful outcome depends upon prompt diagnosis and treatment with intravenous antibiotics and lavage.

Infection with Streptococcus pneumoniae is rare, representing less than $5 \%$ of cases of septic arthritis. ${ }^{1}$ The diagnosis may be difficult if the patient has already received antibiotics. We present the cases of three patients with rheumatoid arthritis and $S$ pneumoniae septic arthritis whose joint infection developed insidiously after a chest infection.

\section{Case reports}

CASE 1

A 48 year old woman with bronchiectasis since childhood developed seronegative rheumatoid arthritis at the age of 36 years in 1973. At the age of 14 years she had two operations to prevent recurrent dislocation of the left patella and a fixation screw persists. When first seen there was synovitis and instability of the left knee. In view of the activity in this joint she had a medical synovectomy with radioactive yttrium in 1973 . By 1984 she was taking 20 units/week of corticotrophin (ACTH) to control her generalised disease activity.

Accepted for publication 20 January 1987

Correspondence to Dr Richard G Hull, Wexham Park Hospital, Slough, Berkshire SL2 4HL.
In early December 1984 she developed a chest infection with increased amounts of purulent spuse tum. One week later she was unable to rise from aN chair and noticed swelling of her left knee. Over the next few days she experienced epigastric pain and frank melaena, which precipitated her admission to hospital.

She was apyrexial and dyspnoeic with widespread rhonchi and coarse crepitations at the left base $\overrightarrow{\vec{P}}$ There were classical longstanding rheumatoid join changes with a warm left knee effusion without other signs of disease activity. The haemoglobin was $86 \mathrm{~g} / \mathrm{l}$, white cell count $9.7 \times 10^{9} / 1$, the erythrocyte sedimentation rate (ESR) was $108 \mathrm{~mm} / \mathrm{h}(8-16 \mathrm{~mm} / \mathrm{h}$ in the previous year). The immunoglobulin levels were $\mathrm{IgG} 4 \cdot 8 \mathrm{~g} / \mathrm{l}$ (normal range (NR) 7.2-19.0 g/l) IgA $0.3 \mathrm{~g} / \mathrm{l}(\mathrm{NR} 0.8-5 \cdot 0 \mathrm{~g} / \mathrm{l})$, and IgM $0.5 \mathrm{~g} / \mathrm{l}$ (NR $0 \cdot 5-2 \cdot 0 \mathrm{~g} / \mathrm{l})$. A chest $x$ ray confirmed bronchiectatice changes. Her knee was aspirated and culture yielded $S$ pneumoniae. Upper gastrointestinal endoscop showed two antral gastric ulcers.

The septic arthritis was treated with intravenous benzylpenicillin for two weeks, followed by oraP amoxycillin for one year and by lavage undef general anaesthetic. The knee was splinted, the pains subsided, and after two weeks she was able to beap weight, using a full length enclosed splint, fitte because of the previous instability of the knee. Shep is now pain free and mobile but has limitation of movement in the knee.

CASE 2

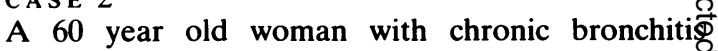
developed seropositive rheumatoid arthritis at the age of 49 years in 1974. In February 1985 shę 
developed a chest infection which was treated with erythromycin and one week later had a generalised increase in joint pain such that she was unable to walk. There was marginally more pain in the right knee. Her general practitioner injected the joint with hydrocortisone, with no improvement. She was admitted as an emergency in March 1985 with a small haematemesis after vomiting consistent with a Mallory-Weiss tear.

On admission she had signs of a right upper lobe pneumonia, confirmed radiologically. There was an effusion of the right knee with reduced range of movement. Investigations showed a haemoglobin of $96 \mathrm{~g} / 1$, white cell count $30.8 \times 10^{9} / 1$, and an ESR of $111 \mathrm{~mm} / \mathrm{h}(18-100 \mathrm{~mm} / \mathrm{h}$ in the previous year). The immunoglobulin levels were IgG $17.4 \mathrm{~g} / \mathrm{l}$, IgA $2.9 \mathrm{~g} / \mathrm{l}$, and $\operatorname{IgM} 1.1 \mathrm{~g} / \mathrm{l}$. Sputum culture showed normal commensals, but aspiration of the right knee yielded Gram positive cocci on film and culture grew $S$ pneumoniae.

The septic arthritis was treated with intravenous flucloxacillin and gentamicin, lavage, and splintage. The antibiotics were changed to intravenous benzylpenicillin when the sensitivities were known, and after two weeks oral amoxycillin was substituted to complete six weeks' total treatment. She was mobilised in a walking splint and discharged eight weeks after admission.

\section{CASE 3}

A 70 year old man with chronic bronchitis developed seropositive rheumatoid arthritis with Sjögren's syndrome at the age of 53 years in 1966. In May 1982 he was admitted to hospital as an emergency with malaise and purulent sputum. He had signs of a right lower lobe pneumonia, which was confirmed radiologically. A sputum stain showed Gram positive diplococci which failed to culture subsequently. He was treated with oxytetracycline and physiotherapy. Six weeks later he was admitted with weakness in both lower limbs. He was unable to walk but did not complain of pain. There were widespread destructive joint changes without signs of inflammation. The knees were not tender but there were small effusions. He was apyrexial.

Investigations showed a haemoglobin of $93 \mathrm{~g} / \mathrm{l}$, white cell count $5.4 \times 10^{9} / \mathrm{l}$, and an ESR of $115 \mathrm{~mm} / \mathrm{h}$ $(31-70 \mathrm{~mm} / \mathrm{h}$ in the previous year). The IgG level was $7 \cdot 1 \mathrm{~g} / \mathrm{l}$, IgA $2 \cdot 9 \mathrm{~g} / \mathrm{l}$, and IgM $2 \cdot 3 \mathrm{~g} / \mathrm{l}$. The sputum continued to show Gram positive diplococci, which failed to grow on culture. Aspiration of the knees showed no fluid in the right knee but on the left pus was aspirated showing Gram positive diplococci on film but again failing to grow on culture. He was treated with intravenous benzylpenicillin but de- veloped erythema multiforme. Treatment was changed to erythromycin for a further four months.

\section{Discussion}

Septic arthritis has a significant morbidity ${ }^{2}$ especially if the diagnosis is delayed or the patient is elderly. Cooper and Cawley reviewed 74 cases of septic arthritis presenting to hospital over a 10 year period up to 1982 and found the $35 \%$ chronic morbidity was avoided in those cases where there was early recognition of infection and appropriate antibiotic therapy. ${ }^{3}$ Established joint disease and immunosupression were other adverse factors. Intra-articular antibiotics and surgical drainage did not confer benefit, though these measures were used in patients already felt to have a poorer outlook for other reasons. The commonest joint involved was the knee in $53 \%$ of cases, and $14 \%$ of patients had rheumatoid arthritis.

Our three patients were elderly, had longstanding rheumatoid arthritis, and at the time of their sepsis had no active disease. None were immunosupressed and one only was receiving ACTH. This patient (case 1) had depressed IgG and IgA levels at the time of the infection, but these had previously been normal. All patients had a history of chronic chest disease with recent infection. None had the high fever and acutely painful swollen joints normally associated with septic arthritis. They all experienced malaise and a generalised increase in joint aches, with marginally more pain in the affected joint. Only one patient (case 2 ) had a polymorphonuclear leucocytosis at $30 \cdot 8 \times 10^{9} / 1$, but all had a recent rise in ESR to a value greater than $100 \mathrm{~mm} / \mathrm{h}$. This is consistent with previous reports. ${ }^{2-4}$ Cooper and Cawley showed a fever of greater than $39^{\circ} \mathrm{C}$ in only $39 \%$ of cases, a leucocytosis greater than $14 \times 10^{9} / 1$ in only $23 \%$, but an ESR of greater than $50 \mathrm{~mm} / \mathrm{h}$ in $60 \%$ and a raised value in most. ${ }^{3}$

$S$ pneumoniae is a rare cause of joint infection. There were no cases in one series of 134 patients from Oxford, ${ }^{2}$ one out of 26 from Taplow, ${ }^{4}$ and one out of 74 from Southampton. ${ }^{3}$ The diagnosis in our patients was confirmed in all by aspiration of the joint. It is of note that blood cultures were negative in all. Gransden et al have reported a series of 325 episodes of pneumococcal bacteraemia, in which septic arthritis occurred in only one case. ${ }^{5}$ In another study of pneumococcal infection in Sweden there were three cases of septic arthritis out of 520 septic episodes. ${ }^{6}$ Eighty one per cent of patients had a predisposing cause for infection, and the largest group was related to alcohol abuse $(32 \%)$. Our patients had no such history and drank infrequently. The presence of a prosthesis or foreign material in a 
joint is well recognised to predispose to infection. One of our patients had a residual bone screw in the lower femur, but well beyond the reflection of the synovium. This was the only surgical implant in our patients.

Sepsis should be considered in a patient with longstanding rheumatoid arthritis if a joint is more painful than usual or if the patient's general condition has deteriorated without obvious cause. Despite prompt treatment all patients lost range of movement and mobility. $S$ pneumoniae infection in particular may be associated with chronic chest disease. We postulate that secondary spread from the lung occurred in our patients. Acute or chronic pulmonary sepsis in a patient with rheumatoid arthritis should alert the physician to the possibility of septic arthritis, especially if the patient remains unwell once the chest infection has been treated. The joints may not appear acutely inflamed. A leucocytosis is often absent and an increase in ESR may be the main clue to the diagnosis.

We are grateful to Dr B M Ansell for allowing us to report patients under her care.

\section{References}

1 Schmid F R. Routine drug treatment of septic arthritis. Clin Rheum Dis 1984: 10: 293-311.

2 Newman J H. Revicw of septic arthritis throughout the antibiotic era. Ann Rheum Dis 1976: 35: 198-205.

3 Cooper C. Cawley M I D. Bacterial arthritis in an English health district: a 10 vear review. Ann Rheum Dis 1986; 45: 458-63.

4 Russell A S. Ansell B M. Septic arthritis. Ann Rheum Dis 1972; 31: $40-4$.

5 Gransden W R. Eykyn S J. Phillips I. Pneumococcal bacter- of aemia: 325 episodes diagnosed at St Thomas`s Hospital. Br Med ō J 1985: 290: 505-8.

6 Burman L A. Norrby R. Trollfors B. Invasive pneumococcal infections: incidence, predisposing factors and prognosis. Rev $\mathrm{O}$ Infect Dis 1985; 7: 133-42. 